\section{Библиографический список}

1. Розенфельд Б. А. Неевклидовы пространства. М. Наука, 1969. 548 с.

2. Ромакина Л. Н. Простые разбиения гиперболической плоскости положительной кривизны // Мат. сб. 2012. Т. 203, вып. 9. С. 83-116.

3. Ромакина Л. Н. Овальные линии гиперболической плоскости положительной кривизны // Изв. Сарат. унта. Нов. сер. Сер. Математика. Механика. Информатика. 2012. Т. 12, вып. 3. С. 37-44.

4. Ромакина Л. Н. Аналоги формулы Лобачевского для угла параллельности на гиперболической плоскости положительной кривизны // Сиб. электрон. мат. изв. 2013. Т. 10. С. 393-407.
5. Ромакина Л. Н. Теорема о площади прямоугольного трехреберника гиперболической плоскости положительной кривизны // Дальневост. мат. журн. 2013. Т. 13, вып. 1. С. 127-147.

6. Ромакина Л. Н. Конечные замкнутые 3(4)-контуры расширенной гиперболической плоскости // Изв. Сарат. ун-та. Нов. сер. Сер. Математика. Механика. Информатика. 2010. Т. 1, вып. 3. С. 14-26.

7. Ромакина Л. Н. Конечные замкнутые 5-контуры расширенной гиперболической плоскости // Изв. Сарат. ун-та. Нов. сер. Сер. Математика. Механика. Информатика. 2011. Т. 11, вып. 1. С. 38-49.

\title{
Hyperbolic Parallelograms of the Plane $\widehat{H}$
}

\section{N. Romakina}

Saratov State University, Russia, 410012, Saratov, Astrahanskaya st., 83, romakinaln@mail.ru

Hyperbolic parallelograms on a Hyperbolic Plane $\widehat{H}$ of the positive curvature in the Cayley-Klein model are investigated. We conducted their classification, obtained the metric correlations between the measure of angles and the expressions of lengths of the edges through a measure of included angles.

Key words: hyperbolic plane $\widehat{H}$ of positive curvature; parallelogram; hyperbolic parallelogram.

\section{References}

1. Rozenfeld B. A. Neevklidovy prostranstva [Non-Euclidean spaces]. Moscow, Nauka, 1969, 548 p. (in Russian). 2. Romakina L. N. Simple partitions of a hyperbolic plane of positive curvature. Sb. Math., 2012, vol. 203, iss. 9. pp. 1310-1341.

3. Romakina L. N. Oval Lines of the Hyperbolic Plane of Positive Curvature. Izv. Sarat. Univ. N.S. Ser. Math. Mech. Inform., 2012, vol. 12, iss. 3, pp. 37-44 (in Russian).

4. Romakina L. N. Analogs of a formula of Lobachevsky for angle of parallelism on the hyperbolic plane of positive curvature. Siberian Electronic Mathematical Reports, 2013, vol. 10, pp. 393-407 (in Russian).
5. Romakina L. N. The theorem of the area of a rectangular trihedral of the hyperbolic plane of positive curvature. Far Eastern Mathematical Journal, 2013, vol. 13, № 1, pp. 127-147 (in Russian).

6. Romakina L. N. Finite Closed 3(4)-Loops of Extended Hyperbolic Plane. Izv. Sarat. Univ. N.S. Ser. Math. Mech. Inform., 2010, vol. 10, iss. 3, pp. 14-26 (in Russian).

7. Romakina L. N. Finite Closed 5-Loops of Extended Hyperbolic Plane. Izv. Sarat. Univ. N.S. Ser. Math. Mech. Inform., 2011, vol. 11, iss. 1, pp. 38-49 (in Russian).

УДК 517.54

\section{К РЕШЕНИЮ НЕОДНОРОДНОЙ КРАЕВОЙ ЗАДАЧИ ГИЛЬБЕРТА ДЛЯ АНАЛИТИЧЕСКОЙ ФУНКЦИИ В МНОГОСВЯЗНОЙ КРУГОВОЙ ОБЛАСТИ В ОСОБОМ СЛУЧАЕ}

\begin{abstract}
Р. Б. Салимов
Доктор фризико-математических наук, профрессор, заведующий касредрой высшей математики, Казанский государственный архитектурно-строительный университет, salimov@5354.ru

Предлагается новый подход к решению краевой задачи Гильберта для аналитической фрункции в многосвязной круговой области, основанный на построении решения соответствующей однородной задачи, когда определяется аналитическая в области функция по известным граничным значениям её аргумента. Рассматривается особый случай задачи, когда индекс задачи неотрицателен и меньше порядка связности области, уменьшенного на единицу. Картина разрешимости задачи зависит от разрешимости и числа решений соответствующей системы линейных алгебраических уравнений.

Ключевые слова: краевая задача Гильберта, индекс задачи, оператор Шварца.
\end{abstract}


Рассматривается применение предложенного в работе [1] подхода к решению краевой задачи Гильберта для аналитической в многосвязной круговой области функции в особом (по терминологии И. Н. Векуа [2, с. 258]) случае, когда индекс задачи неотрицателен и меньше порядка связности области, уменьшенного на единицу. В последнем случае рассматриваемая задача исследована недостаточно полно (это отмечено, в частности, в книге [3, с. 406, 407]).

Для удобства вначале приведем обозначения и используемые в дальнейшем формулы из статьи [1].

Пусть $D$ является $(m+1)$-связной круговой областью, ограниченной полными окружностями $L_{0}, L_{1}, \ldots, L_{m}$ без общих точек, расположенными в плоскости комплексного переменного $z=x+i y$, из которых $L_{0}$ охватывает остальные.

Требуется найти функцию $F(z)=u(z)+i v(z)$, аналитическую и однозначную в области $D$, непрерывно продолжимую на её границу $L=\bigcup_{j=0}^{m} L_{j}$ по краевому условию:

$$
\operatorname{Re}[(a(t)+i b(t)) F(t)]=a(t) u(t)-b(t) v(t)=c(t)
$$

где $a(t), b(t), c(t)$ - заданные на $L$ действительные функции точки $t$ контура $L$, удовлетворяющие условию Гёльдера, - функции класса $H$ на $L$, причем $a^{2}(t)+b^{2}(t) \neq 0$ всюду на $L$.

На $L$ установим положительное направление обхода, при котором область $D$ остается слева. Пусть $t_{j 0}-$ фиксированная точка кривой $L_{j}$. В дальнейшем для функции $f(t)$, заданной на $L_{j}$, под $f\left(t_{j 0}+0\right)$ и $f\left(t_{j 0}-0\right)$ будем понимать пределы, к которым стремится $f(t)$, когда точка $t$ стремится к $t_{j 0}$ соответственно в отрицательном и положительном направлениях.

Краевое условие (1) запишем так:

$$
\operatorname{Re}\left[e^{-i \nu(t)} F(t)\right]=c(t) /|G(t)|
$$

где $G(t)=a(t)-i b(t), \nu(t)=\arg G(t)-$ ветвь, непрерывная всюду на $L$, за исключением, быть может, точек $t_{j 0}$, для которых

$$
\nu\left(t_{j, 0}-0\right)-\nu\left(t_{j, 0}+0\right)=2 \pi \frac{\kappa_{j}}{2},
$$

причем $\kappa_{j} / 2$ - целое число, $j=\overline{0, m}$.

Число $\kappa=\sum_{j=0}^{m} \kappa_{j}$ назовем индексом задачи Гильберта (2), следуя Н. И. Мусхелишвили [4, с. 144] (заметим, что в книгах [2, с. 243; 3, с. 144] индексом этой задачи называется число $\kappa / 2$ ).

Здесь рассмотрим случай, когда $0 \leq \kappa / 2<m$.

1. Обозначим $q_{j}, R_{j}$ соответственно центр и радиус окружности $L_{j}, j=\overline{0, m}$, считая, что $q_{0}=0$, $R_{0}=1$. Примем $t_{00}=1, t_{j 0}=q_{j}+R_{j}, j=\overline{1, m}$. Пусть $t=q_{j}+R_{j} e^{i \gamma}, 0 \leq \gamma<2 \pi$, есть точка окружности $L_{j}$; через $s$ будем обозначать дуговую абсциссу указанной точки кривой $L_{j}$, отсчитываемую от точки $t_{j 0}$ в положительном направлении, $j=\overline{0, m}, s=(2 \pi-\gamma) R_{j}$ при $j=\overline{1, m}$. Под $\arg \left(z-q_{j}\right)$ будем понимать непрерывную ветвь, однозначную в круге $|z|<1$, разрезанном по линии, состоящей из отрезка с концами $z=q_{j}, z=t_{j 0}$ и линии $l_{j}$, лежащей внутри области $D$ и соединяющей точки $t_{j 0}, t_{00}, j=\overline{0, m}$, (при $j=0$ линия $l_{j}$ отсутствует), примем, что эта ветвь на $L_{j}$ принимает значение $\arg \left(t-q_{j}\right)=\gamma, 0 \leq \gamma<2 \pi$.

Пусть $t_{j}=q_{j}+R_{j} e^{i \gamma_{j}}, 0 \leq \gamma_{j}<2 \pi$, есть некоторая точка окружности $L_{j}$, положение этой точки, т. е. число $\gamma_{j}$ будем считать незаданным, $j=\overline{1, m}$.

Пусть $\arg \left(z-t_{j}\right)$ - непрерывная ветвь, однозначная в круге $|z|<1$, разрезанном по линии, состоящей из направленной как $L_{j}$ дуги $t_{j} t_{j 0}$ этой окружности и вышеуказанной кривой $l_{j}$; будем считать, что эта ветвь при $\gamma_{j}>0$ на окружности $L_{j}$, включая точки левого берега разреза по дуге $t_{j} t_{j 0}$, принимает значения

$$
\arg \left(t-t_{j}\right)=\left\{\begin{array}{l}
\left(-\pi+\gamma+\gamma_{j}\right) / 2, \quad 0 \leq \gamma<\gamma_{j}, \\
\left(\pi+\gamma+\gamma_{j}\right) / 2, \quad \gamma_{j}<\gamma<2 \pi, \quad j=\overline{1, m}
\end{array}\right.
$$

При $\gamma_{j}=0$ эта формула принимает вид $\arg \left(t-t_{j 0}\right)=(\pi+\gamma) / 2,0 \leq \gamma<2 \pi$. Поэтому при вышеуказанном выборе ветвей аргументов непрерывная в области $D$ ветвь

$$
\arg \frac{z-t_{j}}{z-q_{j}}=\arg \left(z-t_{j}\right)-\arg \left(z-q_{j}\right)
$$


на $L_{j}$ в случае $\gamma_{j}>0$ принимает значение

$$
\arg \frac{t-t_{j}}{t-q_{j}}=\left\{\begin{array}{lc}
\left(-\pi-\gamma+\gamma_{j}\right) / 2, & 0 \leq \gamma<\gamma_{j} \\
\left(\pi-\gamma+\gamma_{j}\right) / 2, & \gamma_{j}<\gamma<2 \pi
\end{array}\right.
$$

В частности, при $\gamma_{j}=0$ заключаем, что $\arg \frac{z-t_{j 0}}{z-q_{j}}$ на $L_{j}$ принимает значение

$$
\arg \frac{t-t_{j 0}}{t-q_{j}}=\frac{\pi-\gamma}{2}, \quad 0 \leq \gamma<2 \pi
$$

2. Введем в рассмотрение функцию $p_{j}(t)=1$ на вышеуказанной дуге $t_{j} t_{j 0}$ окружности $L_{j}, p_{j}(t)=0$ на остальной части $L_{j}$ и на всех других окружностях - компонентах $L, j=\overline{1, m}$. Далее краевое условие (2) запишем в равносильном виде:

$$
\operatorname{Re}\left[e^{-i \tilde{\nu}(t)} \tilde{F}(t)\right]=\tilde{c}(t),
$$

где

$$
\begin{gathered}
\tilde{\nu}(t)=\nu(t)+\sum_{j=1}^{m}\left[2 \pi p_{j}(t)+2 \arg \frac{t-t_{j}}{t-q_{j}}+\left(1+\kappa_{j} / 2\right) \arg \left(t-q_{j}\right)\right], \\
\tilde{F}(z)=F(z) \prod_{j=1}^{m}\left[\left(z-q_{j}\right)^{1+\kappa_{j} / 2} \cdot\left(\frac{z-t_{j}}{z-q_{j}}\right)^{2}\right], \\
\tilde{c}(t)=\frac{c(t)}{|G(t)|} \prod_{j=1}^{m}\left[\left|t-q_{j}\right|^{1+\kappa_{j} / 2} \cdot\left|\frac{t-t_{j}}{t-q_{j}}\right|^{2}\right] .
\end{gathered}
$$

Будем искать решение $\tilde{F}(z)$ краевой задачи (5) в классе функций, непрерывных в области $D$, вплоть до её границы (при этом, как видно из формулы $(7)$, функция $F(z)$ может иметь особенности в точках $\left.t_{j}, j=\overline{1, m}\right)$. Вначале найдем частное решение $\tilde{F}_{0}(z)$ соответствующей однородной задачи:

$$
\operatorname{Re}\left[e^{-i \tilde{\nu}(t)} \tilde{F}_{0}(t)\right]=0,
$$

отличное от нуля всюду на $L$. Из (9) для аргумента

$$
\arg \tilde{F}_{0}(t)=\tilde{\psi}(t)
$$

такого решения, как и в [1], получим выражение

$$
\tilde{\psi}(t)=\tilde{\nu}(t)+\frac{\pi}{2}+\pi n_{j}
$$

когда $t \in L_{j}$, где $n_{j}-$ произвольное целое число, $j=\overline{0, m}, n_{0}=0$. Таким образом, известны граничные значения $\tilde{\psi}(t)$ функции $\arg \tilde{F}_{0}(z)$ с точностью до слагаемого $\pi n_{j}$ при нефиксированных пока значениях $\gamma_{j}, j=\overline{1, m}$. Остается найти функцию $\tilde{F}_{0}(z)$.

Для нахождения вышеуказанного частного решения $\tilde{F}_{0}(z)$ задачи $(9)$ воспользуемся предложенным в статье [1] подходом. Пусть $z_{0}-$ заданная точка области $D, \arg \left(t-z_{0}\right)$ - граничное значение непрерывной ветви $\arg \left(z-z_{0}\right)$, однозначной в области $D$, разрезанной по линии, лежащей в области $D$ и соединяющей точки $z_{0}, t_{00}$. Как видно из формул $(6),(11)$, функция $\tilde{\psi}(t)-(m+\kappa / 2) \arg \left(t-z_{0}\right)$ непрерывна на каждой из окружностей $L_{j}, j=\overline{0, m}$, поэтому при обходе $L_{j}, j=\overline{0, m}$, приращения не получает. Следовательно, в силу принципа аргумента функция $\tilde{F}_{0}(z) /\left(z-z_{0}\right)^{m+\kappa / 2}$ не имеет нулей в области $D$. Тогда функция

$$
\Phi(z)=-i \ln \left[\tilde{F}_{0}(z) /\left(z-z_{0}\right)^{m+\kappa / 2}\right]
$$

аналитична в области $D$, причем действительная часть её граничного значения равна $\tilde{\psi}(t)-(m+\kappa / 2) \arg \left(t-z_{0}\right)$, т.е. согласно (10), (11) на $L_{j}$

$$
\operatorname{Re} \Phi(t)=\tilde{\nu}(t)+\frac{\pi}{2}+\pi n_{j}-(m+\kappa / 2) \arg \left(t-z_{0}\right), \quad j=\overline{0, m} .
$$


Отсюда в силу (6) с учетом выражений (3) для $\arg \frac{t-t_{j}}{t-q_{j}}$ и $p_{j}(t)$ будем иметь при $t \in L_{j}, j=\overline{1, m}$,

$$
\begin{aligned}
\operatorname{Re} \Phi(t)=\frac{3 \pi}{2} & +\gamma_{j}+\pi n_{j}+\nu(t)+\sum_{j_{1}=1, j_{1} \neq j}^{m}\left(\arg \left(t-q_{j_{1}}\right)+2 \arg \frac{t-t_{j_{1}}}{t-q_{j_{1}}}\right)+ \\
& +\sum_{j_{1}=1}^{m} \frac{\kappa_{j_{1}}}{2} \arg \left(t-q_{j_{1}}\right)-(m+\kappa / 2) \arg \left(t-z_{0}\right),
\end{aligned}
$$

при $t \in L_{0}$ получим:

$$
\operatorname{Re} \Phi(t)=\frac{\pi}{2}+\nu(t)+\sum_{j=1}^{m}\left(2 \arg \frac{t-t_{j}}{t-q_{j}}+\left(1+\kappa_{j} / 2\right) \arg \left(t-q_{j}\right)\right)-(m+\kappa / 2) \arg \left(t-z_{0}\right) .
$$

Поскольку функция $\Phi(z)$ должна быть однозначной и аналитической в области $D$, то должны выполняться условия (см., например, [3, с. 383])

$$
\int_{L} \operatorname{Re} \Phi(t) \alpha_{k}(t) d s=0, \quad k=\overline{1, m}
$$

где $\alpha_{k}(t)=\partial \beta_{k}(t) / \partial n$ есть производная по направлению внутренней для области $D$ нормали в точке $t$ границы $L$ области $D, \beta_{k}(z)$ - гармоническая в области $D$ функция, граничные значения которой определяются формулой

$$
\beta_{k}(t)= \begin{cases}1 & \text { при } t \in L_{k}, \\ 0 & \text { на остальных окружностях, }\end{cases}
$$

$k=\overline{1, m}$. Функции $\beta_{k}(z), \alpha_{k}(t)$ считаем известными, $k=\overline{1, m}$.

Подставляя выражения (13), (14) в условия (15), придем к системе нелинейных уравнений с неизвестными $\gamma_{j}, n_{j}, j=\overline{1, m}$, вопрос о решении которой рассмотрен в работе [1].

Считая, что числа $n_{j}, \gamma_{j}, j=\overline{1, m}$, уже определены, по формулам (13), (14) вычислим $\operatorname{Re} \Phi(t)$ и с помощью оператора Шварца найдем однозначную аналитическую в области $D$ функцию $\Phi(z)=$ $=S(\operatorname{Re} \Phi(t), z)+i v_{0}$, взяв произвольную действительную постоянную $v_{0}=0$.

Тогда согласно (12) будем иметь

$$
\tilde{F}_{0}(z)=e^{i \Phi(z)}\left(z-z_{0}\right)^{m+\kappa / 2}
$$

- частное решение задачи (9), отличное от нуля всюду на $L$. В силу (9) $i e^{-i \tilde{\nu}(t)} \tilde{F}_{0}(t)-$ действительная величина, поэтому условие (5) можно записать так:

$$
\operatorname{Re}\left[\frac{\tilde{F}(t)}{i \tilde{F}_{0}(t)}\right]=c_{*}(t)
$$

где

$$
c_{*}(t)=\tilde{c}(t) /\left(i e^{-i \tilde{\nu}(t)} \tilde{F}_{0}(t)\right) .
$$

Как видно из (17), точка $z_{0}$ является полюсом порядка $m+\kappa / 2$ функции $\tilde{F}(z) /\left(i \tilde{F}_{0}(z)\right)$. Учитывая это, последнюю функцию будем искать в виде

$$
\tilde{F}(z) /\left(i \tilde{F}_{0}(z)\right)=\Psi(z)+i \beta_{0}+\sum_{j=1}^{m+\kappa / 2} \mu_{j} /\left(z-z_{0}\right)^{j},
$$

где $\Psi(z)$ - новая искомая аналитическая и однозначная в области $D$ функция, $\beta_{0}, \mu_{j}-$ произвольные соответственно действительная и комплексная постоянные.

Согласно (18), (20) всюду на $L$ имеем:

$$
\operatorname{Re} \Psi(t)=c_{*}(t)-\sum_{j=1}^{m+\kappa / 2} \operatorname{Re}\left[\mu_{j} /\left(t-z_{0}\right)^{j}\right] .
$$


Функция $\operatorname{Re} \Psi(t)$ должна удовлетворять условиям, аналогичным (15), поэтому должны выполняться соотношения

$$
\sum_{j=1}^{m+\kappa / 2} \int_{L} \operatorname{Re}\left[\mu_{j} /\left(t-z_{0}\right)^{j}\right] \alpha_{k}(t) d s=\int_{L} c_{*}(t) \alpha_{k}(t) d s, \quad k=\overline{1, m}
$$

Эти соотношения представляют собой систему $m$ линейных алгебраических уравнений с $(2 m+\kappa)$ неизвестными $\operatorname{Re} \mu_{j}, \operatorname{Im} \mu_{j}, j=\overline{1, m+\kappa / 2}$. Считая условия (22) выполненными, по значениям $\operatorname{Re} \Psi(t)$ формулы (21) с помощью оператора Шварца находим $\Psi(z)$, тогда в силу (20) будем иметь:

$$
\frac{\tilde{F}(z)}{i \tilde{F}_{0}(z)}=S\left(c_{*}(t)-\sum_{j=1}^{m+\kappa / 2} \operatorname{Re} \frac{\mu_{j}}{\left(t-z_{0}\right)^{j}}, z\right)+i \beta_{0}+\sum_{j=1}^{m+\kappa / 2} \frac{\mu_{j}}{\left(z-z_{0}\right)^{j}} .
$$

Отсюда, принимая во внимание (17) и выражение $(7)$ для $\tilde{F}(z)$, получим:

$$
\begin{aligned}
F(z) & =i e^{i \Phi(z)}\left(z-z_{0}\right)^{m+\kappa / 2}\left\{S\left(c_{*}(t)-\sum_{j=1}^{m+\kappa / 2} \operatorname{Re} \frac{\mu_{j}}{\left(t-z_{0}\right)^{j}}, z\right)+i \beta_{0}+\right. \\
& \left.+\sum_{j=1}^{m+\kappa / 2} \frac{\mu_{j}}{\left(z-z_{0}\right)^{j}}\right\} /\left(\prod_{j=1}^{m}\left[\left(z-q_{j}\right)^{1+\kappa_{j} / 2} \cdot\left(\frac{z-t_{j}}{z-q_{j}}\right)^{2}\right]\right) .
\end{aligned}
$$

Так как мы ищем решение $F(z)$ краевой задачи (2), непрерывное на $L$, то мы должны потребовать, чтобы выражение в фигурных скобках (т. е. выражение (23)) последней формулы обращалось в нуль второго порядка в точках $t_{j_{1}}, j_{1}=\overline{1, m}$. С этой целью потребуем, чтобы выполнялось соотношение

$$
\operatorname{Im} S\left(c_{*}(t)-\sum_{j=1}^{m+\kappa / 2} \operatorname{Re} \frac{\mu_{j}}{\left(t-z_{0}\right)^{j}}, t_{j_{1}}\right)+\beta_{0}+\sum_{j=1}^{m+\kappa / 2} \operatorname{Im} \frac{\mu_{j}}{\left(t_{j_{1}}-z_{0}\right)^{j}}=0, \quad j_{1}=\overline{1, m}
$$

кроме того, указанное выражение $(23)$, заменив в нем предварительно $t$ на $t_{*} \in L$, запишем для $z=t=\left(q_{j_{1}}+R_{j_{1}} e^{i \gamma}\right) \in L_{j_{1}}$, далее потребуем, чтобы производная по $\gamma$ от него в точке $t_{j_{1}}$ обращалась в нуль, и будем иметь:

$$
\begin{gathered}
\lim _{\gamma \rightarrow \gamma_{j_{1}}} \frac{\operatorname{Im}\left[S\left(c_{*}\left(t_{*}\right), t\right)-S\left(c_{*}\left(t_{*}\right), t_{j_{1}}\right)\right]}{\gamma-\gamma_{j_{1}}}- \\
-\sum_{j=1}^{m+\kappa / 2}\left\{\left.\operatorname{Im}\left[S\left(\operatorname{Re} \frac{\mu_{j}}{\left(t_{*}-z_{0}\right)^{j}}, t\right)\right]_{\gamma}^{\prime}\right|_{\gamma=\gamma_{j_{1}}}+\operatorname{Im} \frac{\mu_{j} j R_{j_{1}} e^{i \gamma_{j_{1}}}}{\left(t_{j_{1}}-z_{0}\right)^{j+1}}\right\}=0,
\end{gathered}
$$

здесь $j_{1}=\overline{1, m}$. Последние условия $(25),(26)$ являются необходимыми для непрерывности функции $F(z)$ формулы (24) в точках $t_{j_{1}}$; применяя результаты статьи [5] к функции (23), с учетом (8), (19) можно показать, что они являются так же достаточными и попутно убедиться в существовании выражения в левой части уравнения (26).

Соотношения (22), (25), (26) представляют собой систему линейных $3 m$ уравнений с $\kappa+2 m+1$ действительными неизвестными $\operatorname{Re} \mu_{j}, \operatorname{Im} \mu_{j}, \beta_{0}$.

При выполнении условий (22) оператор Шварца в формулах (23)-(26) определяет однозначную функцию и представляет собой интеграл по кривой $L$ с плотностью $c_{*}(t)-\sum_{j=1}^{m+\kappa / 2} \operatorname{Re}\left[\mu_{j} /\left(t-z_{0}\right)^{j}\right]$ и однозначным ядром [3, с. 384].

Не приводя явных выражений, будем считать, что в системе уравнений (22), (25), (26) коэффициенты при неизвестных $\operatorname{Re} \mu_{j}, \operatorname{Im} \mu_{j}, j=\overline{1, m}$, уже вычислены. Отметим лишь, что в уравнении (25) коэффициент при неизвестном $\operatorname{Re} \mu_{j}$ равен значению суммы

$$
\operatorname{Im} S\left(-\operatorname{Re} \frac{1}{\left(t_{*}-z_{0}\right)^{j}}, t\right)+\operatorname{Im} \frac{1}{\left(t-z_{0}\right)^{j}}
$$


в точке $t=t_{j 1}$, в уравнении (26) - значению производной по $\gamma$ от последней суммы при $\gamma=\gamma_{j 1}$, когда $t=q_{j 1}+R_{j 1} e^{i \gamma}$, причем существование указанной производной от первого слагаемого последней суммы следует из результатов, приведенных в книге [3, с. 43].

Пусть $0 \leq \kappa<m-1$ и число неизвестных $\kappa+2 m+1<3 m-$ числа уравнений системы (22), (25), (26). Обозначим ранг основной матрицы этой системы через $r$. В рассматриваемом случае $r \leq \kappa+2 m+1$. Совместность системы (22), (25), (26) равносильна совпадению рангов основной и расширенной матриц системы. Это совпадение будет иметь место, если все миноры $(r+1)$-го порядка расширенной матрицы системы, содержащие базисный минор основной матрицы и свободные члены системы, обращаются в нуль. Ясно, что число таких миноров равно $3 m-r$. Приравняя нулю эти миноры, получаем $3 m-r$ условий совместности системы, которые представляют собой условия разрешимости задачи (2). При выполнении этих $3 m-r$ условий совместности система (22), (25), (26) равносильна системе $r$ её уравнений, основная матрица которой имеет ранг $r$, причем один из миноров этой матрицы является базисным минором основной матрицы исходной системы.

При $r=\kappa+2 m+1$ последняя система $r$ уравнений с $r$ неизвестными имеет единственное решение, которому отвечает единственное решение (24) задачи (2); оно существует при выполнении $3 m-r=m-1-\kappa$ условий разрешимости.

При $r<\kappa+2 m+1$ вышеуказанная система $r$ уравнений содержит $\kappa+2 m+1$ неизвестных. Из этой системы $r$ неизвестных, коэффициенты которых являются элементами базисного минора (основной матрицы системы (22), (25), (26)), выразим через $\kappa+2 m+1-r$ остальных неизвестных, значения которых могут быть выбраны произвольно. Подставляя найденные выражения в формулу (24), получим зависящее от $\kappa+2 m+1-r$ произвольных действительных постоянных решение задачи (2), которое существует при выполнении $3 m-r$ условий разрешимости. Итак, имеет место

Теорема 1. Если $0 \leq \kappa<m-1$ и выполняются $3 m-r$ условий совместности системы (22), (25), (26), то при $r=\kappa+2 m+1$ задача (2) имеет единственное решение согласно (24), при $r<\kappa+2 m+1$ решение задачи (2) определяется формулой (24) и содержит $\kappa+2 m+1-r$ произвольных действительных постоянных.

При $\kappa=m-1$ число неизвестных $\kappa+2 m+1=3 m$, т. е. равно числу уравнений системы (22), (25), (26). Если $r=3 m$, то система совместна и имеет единственное решение, тогда, подставляя найденные значения неизвестных, по формуле (24) определим единственное решение задачи (2).

Если $r<3 m$, то система (22), (25), (26) будет совместна при выполнении $3 m-r$ условий, записываемых, как указано выше; при их выполнении исходная система решается так же, как и в предыдущем случае при $r<\kappa+2 m+1$, если число $\kappa+2 m+1$ в тексте заменить на $3 m$. Следовательно, справедлива

Теорема 2. Пусть $\kappa=m-1$. Если $r=3 m$, то задача (2) имеет единственное решение, определяемое формулой (24). Если $r<3 m$ и выполняются $3 m-r$ условий совместности системь (22), (25), (26), то решение задачи (2) определяется формулой (24) и содержит $3 m-r$ действительньх произвольных постоянных.

Рассмотрим случай $\kappa \geq m(\kappa<2 m)$. Тогда число неизвестных $\kappa+2 m+1>3 m-$ числа уравнений системы (22), (25), (26). Если $r=3 m$, то эта система совместна, так как ранг её расширенной матрицы равен $3 m$, и рассматриваемая система имеет решение, зависящее от $\kappa+2 m+1-3 m=\kappa-m+1$ произвольно задаваемых неизвестных, через которые выражаются неизвестные с коэффициентами, равными элементам базисного минора вышеуказанной системы. Решение задачи (2) согласно формуле (24) зависит от $\kappa-m+1$ вышеназванных действительных произвольных постоянных.

Если $r<3 m$, то система (22), (25), (26) совместна, если выполняются $3 m-r$ условий совместности - условий обращения в нуль всех миноров $(r+1)$-го порядка расширенной матрицы системы, содержащих базисный минор основной матрицы и свободные члены системы. Исходная система равносильна системе $r$ её уравнений, основная матрица которой содержит вышеуказанный базисный минор. Из последней системы $r$ неизвестных с коэффициентами, являющимися элементами базисного минора, выразим через $\kappa+2 m+1-r$ остальных неизвестных, значения которых могут быть заданы произвольно. При этом формула (24) дает решение задачи (2), зависящее от $\kappa+2 m+1-r$ действительных произвольных постоянных. Итак, справедлива

Теорема 3. Пусть $\kappa \geq m(\kappa<2 m)$. Если $r=3 m$, то задача (2) имеет решение (24), зависящее от $\kappa-m+1$ действительных произвольных постоянных. Если $r<3$ и выполняется $3 m-r$ 
условий совместности системы (22), (25), (26), то задача (2) имеет решение (24), зависящее от $\kappa+2 m+1-r$ действительных произвольных постоянных.

В теореме 1 и при $r<3 m$ в теоремах 2,3 предполагается, что выполняются $3 m-r$ условий совместности системы (22), (25), (26); кроме того, принимается, что при $r<\kappa+2 m+1$ в теореме 1 и при $r<3 m$ в теоремах 2,3 обращаются в нуль все миноры $(r+1)$-го порядка основной матрицы системы (22), (25), (26), содержащие базисный минор этой матрицы, с общим числом, равным соответственно $(3 m-r)(\kappa+2 m+1-r),(3 m-r)^{2},(3 m-r)(\kappa+2 m+1-r)$. Ясно, что эти предположения являются условиями разрешимости задачи (2), обеспечивающими реализацию соответствующих условий рассматриваемой теоремы, и могут выполняться только в исключительных случаях.

В книге [2, с.274,275] приведен пример, для которого $c(t) \equiv 0$ всюду на $L$, и упомянутые исключительные случаи реализуются, когда краевая задача (2) имеет ненулевые решения при $0 \leq \kappa / 2 \leq m-1$ (в наших обозначениях).

Вопросы совместности совокупности всех указанных условий в любой из теорем требуют особого рассмотрения в каждом конкретном случае с учетом того, что коэффициенты и свободные члены системы (22), (25), (26) зависят от формы области $D$ и свойств заданных функций $\nu(t), c(t)$ краевого условия (2).

Уместно отметить, что условия совместности системы (22), (25), (26) выполняются автоматически, если $c(t) \equiv 0$ всюду на $L$.

Работа выполнена при финансовой поддержке РФФИ (проект 12-01-00636-а).

\title{
Библиографический список
}

1. Салимов Р. Б. Модификация нового подхода к решению краевой задачи Гильберта для аналитической функции в многосвязной круговой области // Изв. Сарат. ун-та. Нов. сер. Сер. Математика. Механика. Информатика. 2012. Т. 12, вып. 1. С. 32-38.

2. Векуа И. Н. Обобщенные аналитические функции. М. : Физматгиз, 1959. 628 с.
3. Гахов Ф. Д. Краевые задачи. М. : Наука, 1977. 640 с. 4. Мусхелишвили Н. И. Сингулярные интегральные уравнения. М. : Наука, 1968. 511 с.

5. Салимов Р. Б. Некоторые свойства аналитических в круге функций и их приложения к исследованию поведения сингулярных интегралов // Изв. вузов. Математика. 2012. № 3. С. 42-50.

\section{To a Solution of the Inhomogeneous Riemann-Hilbert Boundary Value Problem for Analytic Function in Multiconnected Circular Domain in a Special Case}

\begin{abstract}
R. B. Salimov
Kazan State University of Architecture and Engineering, Russia, 420043, Kazan, Zelenaya st., 1, salimov@5354.ru

The author offers a new approach to solution of the Riemann-Hilbert boundary value problem for analytic function in multiconnected circular domain. This approach is based on construction of solution of corresponding homogeneous problem, when analytic in domain function is being defined by known boundary values of its argument. The author considers a special case of a problem when the index of a problem is more than zero and on unit of less order of connectivity of domain. Resolvability of a problem depends on number of solutions of some system of the linear algebraic equations.
\end{abstract}

Key words: Riemann-Hilbert boundary value problem, index of a problem, Schwarz's operator.

\section{References}

1. Salimov R. B. Modification of new approach to solution of the Hilbert boundary value problem for analytic function in multi-connected circular domain. Izv. Saratov. Univ. N.S. Ser. Math. Mech. Inform., 2012, vol. 12, iss. 1, pp. 32-38 (in Russian).

2. Vekua I. N. Generalized analytic functions. Oxford, Pergamon Press, 1962, 668 p. (Rus. ed.: Vekua I. N. Obobshchennye analiticheskie funktsii. Moscow, Fizmatgiz, 1959, 628 p.)

3. Gahov F. D. Boundary-Value Problems. Moscow, Nauka, 1977, 640 p. (in Russian).
4. Muskhelishvili N. I. Singular Integral Equations. Boundary-Value Problems of the Theory of Functions and Some of Their Applications to Mathematical Physics. Moscow, Nauka, 1968, 511 p. (in Russian).

5. Salimov R. B. Some properties of analytic in a disc functions and their applications to study of behaviour of singular integrals. Russian Math. (Izvestiya VUZ. Matematika), 2012, vol. 56, no. 3, pp. 36-44. 